\title{
Incentivos regulatorios para el desarrollo de la energía eólica marina en Chile
}

\section{Regulatory Incentives for Develop Offshore Wind Energy in Chile}

Alberto Olivares Gallardo*

El trabajo analiza los principales esquemas normativos utilizados por los diversos países para promover la energía eólica marina en sus costas. Ello, con la finalidad de identificar aquellas medidas que pueden ser implementadas en Chile para integrar esta tecnología a su matriz energética, que es vista como una de las fuentes energéticas del futuro.

Palabras clave: Energías renovables; energía eólica marina; incentivos regulatorios.
This paper analyzes the main regulatory schemes used by the various countries to encourage offshore wind energy projects on their coasts. This, in order to identify those measures that can be implemented in Chile to incorporate this technology into its energy mix, which is seen as one of the energy sources of the future.

Keywords: Renewable energies; offshore wind energy; regulatory incentives.

\section{Introducción}

Hasta hace unos pocos años la energía eólica marina era una fuente energética restringida a unos pocos países desarrollados con una fuerte conciencia ambiental, que podían permitirse incorporar a este recurso renovable para producir electricidad a unos costes muy elevados. El incipiente desarrollo tecnológico también limitaba la expansión de esta fuente energética a suelos marinos poco profundos.

\footnotetext{
* Doctor en Derecho por la Universidad Rovira i Virgili (España). Investigador contratado de proyecto, Centro de Estudios Avanzados de Blanes, Consejo Superior de Investigaciones Científicas(CEAB-CSIC). Número ORCID: 0000-0003-3294-4658. Correo electrónico: a.olivares@ceab.csic.es. Dirección postal: Carretera d'accés a la Cala Sant Francesc 14, Blanes, 17300, España.
}

Artículo recibido el 10 de mayo de 2021 y aceptado el 15 de junio de 2021. 
Por ello, no resulta extraño que la explotación de la energía eólica marina se haya iniciado en los países ribereños de los mares del Norte y Báltico. Dinamarca, Suecia, Noruega, Alemania y Países Bajos comenzaron a impulsar la energía eólica marina en los años 90, que posteriormente se expandiría a otros países, a medida que el desarrollo tecnológico iba dando soluciones a los problemas derivados de su alto coste y las limitaciones técnicas, hasta llegar al estado de expansión actual.

Hoy podemos encontrar también parques eólicos marinos funcionando, en ejecución o en cartera, en diversos países de Europa, Asia, Oceanía y Norteamérica ${ }^{1}$.

La reducción de costes y los avances tecnológicos en el diseño de los aerogeneradores y de las redes eléctricas marinas, han permitido a esta industria ir derribando la mayor parte de las barreras técnicas y económicas que limitaban su crecimiento, para situarse como una de las fuentes energéticas del futuro. Al mismo tiempo, los países han ido incorporando diversos esquemas jurídicos para el incentivo de la eólica marina, que han propiciado un marco normativo favorable para la inversión de esta industria energética.

Este escenario optimista es confirmado por la International Energy Agency (IEA), que proyecta unos $560 \mathrm{GW}$ de capacidad instalada en energía eólica marina para 2040, posicionando al sector como una industria relevante de la economía mundial, que moverá más de un billón de dólares en negocios ${ }^{2}$.

Sin embargo, esta fuente energética sigue lejos de las costas latinoamericanas. Aunque el Banco Mundial ha cifrado el potencial técnico de la región en $6300 \mathrm{GW}^{3}$, solo Brasil ha anunciado recientemente un proyecto para incorporar a esta tecnología en su sistema eléctrico, a través de un conjunto de parques eólicos offshore que se instalarían en el norte del país ${ }^{4}$.

En el caso de Chile, Ilama especialmente la atención que no existan proyectos para implementar parques eólicos marinos, a pesar de tener más de $6.000 \mathrm{~km}$. de costa, de haber experimentado un crecimiento exponencial de las energías renovables en los últimos $a_{n ̃ o s}{ }^{5}$, y de tener un potencial técnico para instalar $957 \mathrm{GW}$ de energía eólica offshore ${ }^{6}$.

\footnotetext{
1 WIND EUROPE 2019, 7-8.

2 International Energy Agency 2019-a, 14-33. Debe aclararse que el informe de la IEA habla de un negocio de "1 trillion dollars", es decir, 1 billón de dólares para nuestro idioma.

3 World Bank \& Energy Sector Management Assistance 2020. Además, el mismo informe destaca el potencial técnico de Argentina (1870 GW), Brasil (1228 GW), Chile (957 GW), Perú (347 GW) y Uruguay (275 GW).

4 Puede revisarse la nota periodística sobre los proyectos de energía eólica offshore "Primeros pasos de la eólica marina en Brasil".

${ }^{5}$ En este sentido, un estudio reciente sitúa a Chile como el país emergente más atractivo para invertir en energías limpias. Bloomberg Nef 2020, 6.

6 World Bank \& Energy Sector Management Assistance Program 2020.
} 
De esta forma, el presente trabajo tiene por objetivo analizar aquellos aspectos relevantes de los esquemas normativos generados por los países para incentivar la energía eólica marina. Ello, con la finalidad de proyectar en el país un entorno normativo favorable para la implementación de parques eólicos offshore.

Como se verá, la inclusión de la energía eólica marina no solo busca aumentar la capacidad instalada de las energías renovables para reemplazar a las energías convencionales, sino que también forma parte de la estrategia que los países han ido adaptando para cumplir con los compromisos internacionales asumidos para la transición hacia una Economía neutra en carbono7.

En este contexto, un adecuado esquema regulatorio incentiva a las empresas eléctricas que operan en un país a invertir en proyectos de eólica marina, una fuente energética renovable que tendrá un rol esencial en la lucha contra el cambio climático ${ }^{8}$.

El estudio se estructura en tres apartados principales en que se analiza a las energías renovables como parte de la Acción por el Clima; el desarrollo de la energía eólica marina y; las diversas normativos adoptados por diferentes países para fomentar esta fuente energética en sus costas.

\section{Las energías renovables y el cambio climático}

En el presente siglo, la Acción por el Clima se ha tomado la agenda ambiental, obligando a los países a asumir mayores compromisos de reducción de emisiones de gases de efecto invernadero, elaborar estrategias de mitigación y adaptación al cambio climático, y transitar hacia un modelo de desarrollo sostenible, neutro en carbono?. La actividad económica de los países debe ser compatible con la estrategia climática global ${ }^{10}$.

Ello incluye también al sector energético que, no obstante, ya venía experimentando desde la última década del siglo pasado, un importante proceso de transformación de su matriz energética, con una progresiva integración de las energías renovables -especialmente con la solar y la eólica-, que para 2010 solventaba el 17\% del consumo total de la energía final en el mundo ${ }^{11}$.

No obstante, la Acción por el Clima obliga a dar un paso más en la transformación de sus matrices energéticas. Ya no basta con reducir el consumo de energías fósiles reemplazándolas con energías renovables. Los países,

\footnotetext{
7 Sobre esta idea, ACUERdo de París 2015.

8 Commission Europea 2020-a, 1-3.

9 En este sentido, el Objetivo de Desarrollo 13, de La Agenda 2030 para el Desarrollo Sostenible, denominada "Acción por el Clima", busca que los países se adapten al cambio climático y logren un desarrollo bajo en carbono. Véase PROGRAMA DE NACIONES LAS NACIONES UNIDAS PARA EL DeSARROLlo 2020.

10 European Commission 2018, 3-6.

11 WORLD BANK 2021.
} 
deben aumentar sus esfuerzos, y transitar hacia un modelo energético hipocarbónico, centrado en energías limpias ${ }^{12}$.

La transición energética, "proceso de transformación de las estructuras económica, técnica y socioambiental de la actividad energética para adaptarse a los nuevos desafíos globales y locales, siendo el más importante de ellos el cambio climático"13, representa un cambio de paradigma que nos obliga a reformular la relación sociedad-energía, adaptándola a la estrategia global por el clima.

Comenzamos a dejar atrás un modelo energético centralizado y contaminante ${ }^{14}$, para avanzar hacia otro, neutro en carbono, caracterizado por la promoción de una gestión descentralizada, con un fuerte componente de generación distribuida y de autoconsumo, y donde el desarrollo tecnológico juega un rol esencial.

Dos elementos centrales de la transición energética. En el primer lugar, el foco está puesto en la reorientación del consumo energético mundial hacia la electricidad, como principal fuente energética del siglo XXI. Desde esta perspectiva, las principales actividades intensivas en consumo de energía -transporte y movilidad, minería, industria, etc.-, han iniciado un proceso de conversión progresiva hacia la electrificación de sus procesos.

En segundo término, la generación de energía eléctrica debe ser climáticamente neutra en el horizonte $2050^{15}$. En este escenario, las energías renovables asumen un rol principal como motor de la transición hacia un nuevo modelo energético neutro en carbono y centrado en la electricidad.

Es alrededor de esta dualidad electricidad-energías renovables, que comienza a diseñarse el proceso de descarbonización de la matriz energética mundial, para transitar hacia un modelo energético poscarbono que, debe advertirse, todavía no está totalmente definido. Sus alcances están condicionados por el desarrollo tecnológico. Así por ejemplo, los avances en almacenamiento energético determinarán si la flexibilidad del sistema eléctrico podrá ser solventada por las propias renovables, o requerirá de otras tecnologías de respaldo -como el gas natural o la energía nuclear- para dar seguridad al sistema ${ }^{16}$.

\footnotetext{
12 De acuerdo con el Parlamento Europeo 2018, el sector de la energía es el responsable del 80\% de las emisiones de gases de efecto invernadero. En el mismo sentido, la International Energy Agency señala que las emisiones globales de CO2 relacionadas con la energía en 2018 fueron de 33,1 Gt CO2. International Energy Agency 2019-c, 4.

13 Olivares 2019, 566.

14 Sobre el modelo energético dominante, véase: Rivero 2011; PAREJo 2010; Ariño 2006; PolutT 1999.

15 European Commission 2018, 6.

16 En este sentido, la Unión Europea ha proyectado para 2050 un escenario en que la columna vertebral del sistema eléctrico europeo libre de carbono tendrá una matriz con $80 \%$ de fuentes de energía renovable (cada vez más localizadas en alamar) y $15 \%$ de energía nuclear. EUROPEAN COMMISSION 2018, 9.
} 
No obstante, existen ciertos puntos de encuentro, determinados por la obligación de transitar hacia una matriz neutra en carbono: i) la electrificación de la matriz energética; ii) las energías renovables como principal fuente de generación eléctrica, y; iii) un intenso desarrollo tecnológico centrado en un conjunto de tecnologías disruptivas, como el almacenamiento o el hidrógeno verde, que aumentan la flexibilidad de las energías renovables.

En este contexto, la transición energética se plantea como un proceso de transformación mayor del sector, para adaptarse al cambio climático. De esta forma, las energías renovables dejan de ser un objetivo propio de política energética y pasan a ser uno de los más importantes instrumentos de mitigación del cambio climático. La transición hacia un modelo energético poscarbono obliga a los países a incorporar nuevos desafíos referidos al desarrollo de las energías renovables.

No obstante, esta transición opera en dos velocidades distintas: una impulsada por el propio sector -industria y regulador-, y otra promovida desde la agenda de cambio climático.

Por una parte, el marco regulatorio propiamente energético -poco sensible a los cambios-, ha ido adaptando progresivamente la estructura del sistema energético y de su mercado, eliminando las barreras normativas, técnicas y económicas para la plena incorporación de las energías renovables al mercado de generación eléctrica. En este sentido, ha operado un cierto continuismo del modelo; es decir, el regulador y la industria energética han entendido que debe transitarse hacia un sistema energético neutro en carbono, liderado por la electricidad y las energías renovables. Para ello, han ido adoptando esquemas regulatorios y acuerdos para acelerar el reemplazo de las centrales más contaminantes por energías renovables, pero sin afectar de manera esencial el modelo vigente ${ }^{17}$.

Por otra parte, la Acción por el Clima presiona por cambios estructurales de fondo en el modelo energético, que impacten positivamente en la reducción de emisiones de gases de efecto invernadero de los países. Ello implica comenzar a descentralizar la gestión, promoviendo la generación distribuida; entregar un nuevo rol del consumidor como pequeño productor; el uso de redes inteligentes; el impulso a nuevas tecnologías disruptivas; pensar en diseñar un mercado de estructura dual, integrado por el mercado eléctrico vigente y otro para estas nuevas formas de generación que operan en pequeños sistemas, etc. ${ }^{18}$.

De esta forma, al amparo de ambos marcos normativos, se ha ido desarrollando un nuevo modelo energético, que intenta conciliar dos visiones

\footnotetext{
17 Otras fuentes renovables, como la geotermia o la biomasa, tienen todavía un aporte residual en la matriz energética mundial, aunque especialmente la biomasa está aumentando su aporte energético en algunos países como Noruega, Dinamarca o Alemania, principalmente a través de la calefacción distrital. Véase: WERNER 2017.

18 Olivares 2019, 570-574; European Commission 2018, 2-7.
} 
distintas de lo que debe ser la transición energética. En este contexto, comienzan a aparecer nuevas fuentes energéticas de transición, que han nacido bajo la lógica y las estructuras actuales de la industria energética, pero que tienen una vocación transformadora de la actividad energética. Impulsan la descentralización de la gestión; son complementarias a las tecnologías de almacenamiento e hidrógeno verde; promueven el empleo de calidad y el desarrollo tecnológico de los países ${ }^{19}$, y; disminuyen la tensión socioambiental en el territorio ${ }^{20}$. Una de ellas es la energía eólica marina, que revisaremos a continuación.

\section{La energía eólica marina}

Es posible distinguir dos fases en el desarrollo de la energía eólica marina. En una primera etapa, que va desde que se inicia la explotación comercial de este recurso energético hasta finales de la primera década de este siglo, podemos observar la etapa más compleja del proceso de aprendizaje de esta nueva tecnología, que le permitirá comenzar a reducir costes y proyectar su futura competitividad económica.

En una segunda fase, que se inicia a continuación -alrededor de 2010-y se extiende hasta hoy, la eólica offshore comienza el despegue como fuente energética relevante para la transición energética y, consecuentemente, para la Acción para el Clima. Abandona su zona de confort -el norte de Europapara comenzar a expandirse en las costas de Asia y Norteamérica.

Revisaremos, a continuación, el proceso de desarrollo que ha tenido la energía eólica marina, siguiendo este esquema.

\section{Primera etapa. Inicio y crecimiento}

El primer parque eólico marino comercial -todavía en funcionamiento-, se instaló en las costas danesas de Vindeby, en el año 1991. Con una capacidad de $5 \mathrm{MW}^{21}$, aportada por 11 aerogeneradores que ofrecían una potencia de $450 \mathrm{~kW}$ cada uno, fue ubicado a $2,5 \mathrm{~km}$. de distancia de la costa y a una profundidad de entre 2,5 y $5 \mathrm{mts}$. del nivel del $\mathrm{mar}^{22}$. Siguieron a este proyecto nuevas iniciativas que se concretaron en las costas de Suecia, Países Bajos, Reino Unido, Alemania e Irlanda, que posicionaron a Europa como exclusivo promotor de esta fuente energética.

Para 2005, las costas del norte del continente europeo acogían unos veinte proyectos, que sumaban una capacidad instalada de $695 \mathrm{MW}$. Los avances tecnológicos permitían a los aerogeneradores alcanzar capacidades de hasta 3 MW. En 2010, existían más de cuarenta parques eólicos marinos,

\footnotetext{
19 La creación de empleos de calidad y el desarrollo tecnológico como objetivos del impulso a las energías renovables, y en especial de la eólica marina, véase COMISIÓN EUROPEA 2020-a, 2-5.

20 Sobre esta idea, COMIIIÓN EUROPEA 2020-b, 9.

21 Olivares 2021, 571; Comisión Europea 2020-b, 1.

22 Diaz 2016, 3; Rodriguez, Pereira y Toval 2011, 6.
} 
que aportaban en total más de $3 \mathrm{GW}^{23}$. Asimismo, se sumaban nuevos países que incorporaban esta tecnología a sus combinaciones energéticas, como Bélgica, Finlandia y China ${ }^{24}$.

Desde una perspectiva institucional, también en esta primera fase se comienza a articular, por la Unión Europea, un plan de acción para el incentivo a la industria eólica marina, acompañada de un fuerte incentivo a la innovación y la investigación de esta tecnología. En este sentido, el año 2001, en colaboración con algunos estados miembros y empresas del sector, la UE implementó la "Acción concertada sobre energía eólica offshore en Europa" (CA-OWEE/RTD), que sería la primera medida adoptada para consensuar una estrategia común de promoción de la energía eólica marina ${ }^{25}$.

A partir de esta instancia colaborativa, la Unión Europea comienza a generar una importante cantidad de información relativa a este recurso energético y a desplegar fuertes incentivos para la investigación en nuevas tecnologías, que permitiesen a la eólica offshore alcanzar una reducción de sus costes que la hicieran competitiva. Los principales lineamientos de una estrategia para las energías marinas son recogidos en el Libro Verde "Hacia una futura política marítima de la Unión Europea: perspectiva europea de los océanos y los mares", de 2006, que remarca el interés energético de los océanos y la necesidad de avanzar en su explotación, como parte de una estrategia energética sostenible, que además genere crecimiento económico y empleo para las zonas $\operatorname{costeras}^{26}$.

Posteriormente, esta cuestión sería desarrollada por la Comisión Europea en la Comunicación de la Comisión Europea de 2008, sobre energía eólica marina, que incluiría el fomento a esta, como parte de las acciones necesarias para alcanzar los objetivos de política energética para el año $2020^{27}$.

Como vemos, hasta 2010 -esto es, en sus primeros veinte años de explotación comercial-, hubo un importante desarrollo tecnológico en torno a la energía eólica marina, aunque su crecimiento, si bien progresivo, en realidad fue moderado, manteniéndose como una tecnología exclusivamente europea, y más exactamente, restringida al norte de este continente.

Esta primera fase de desarrollo de esta tecnología está ligada al proceso de impulso que están teniendo las energías renovables en general, aunque se reconoce desde un primer momento por la UE, las características económicas y técnicas especiales que la hacen avanzar a marchas distintas de

\footnotetext{
23 European Wind Energy Association 2011, 14.

24 Puede verse una relación de los diversos proyectos impulsados en estos países, así como de otros que se sumaron progresivamente a la construcción de parques eólicos marinos hasta 2012 en: Rodríguez, Pereira y Toval 2011, 6.

25 Henderson 2002, 17.

26 SANZ 2007, 906-911.

27 Díaz 2016, 6; Comisión Europea 2008.
} 
las otras fuentes renovables ${ }^{28}$. En este sentido, la propia Comisión Europea reconocía que "comparada con la energía eólica terrestre, la marítima es más compleja y costosa de instalar y mantener", aunque estimaba que "las instalaciones marítimas irán ganado importancia" 29 .

De esta forma, esta etapa se centra en la reducción de costes, el desarrollo tecnológico de la infraestructura -aerogeneradores y redes marinas-, y la consolidación de una estrategia europea común, para avanzar hacia una fase siguiente de expansión global.

\section{Segunda etapa. Despegue y expansión}

A partir de 2010, la estrategia para la eólica marina recibe un nuevo impulso e inicia un proceso de fuerte crecimiento, que incluye el desarrollo de esta tecnología fuera de las costas de Europa. China pone en funcionamiento sus tres primeros parques eólicos offshore, iniciativa que pronto se replicaría en otros países de Asia y Norteamérica.

Uno de los elementos centrales de esta nueva estrategia de desarrollo es el impulso de una red eléctrica marina para conectar los numerosos proyectos de energía eólica marina a las redes eléctricas nacionales del norte de Europa $^{30}$. En diciembre de 2009 diversos países de la Unión Europea más Noruega, suscriben un memorando de entendimiento para crear la Iniciativa de Redes Marinas de los países del Mar del Norte ${ }^{31}$. Este acuerdo busca desarrollar de manera coordinada redes marinas de transporte de electricidad, uno de los puntos clave en el desarrollo de esta fuente energética. Además, en 2010 la Comisión Europea publicaría su relevante Comunicación "Las prioridades de infraestructura energética a partir del 2020. Esquema para una red de energía europea integrada"32, que puso a la red marina en el centro de la política energética de la Unión Europea.

Por otra parte, comenzó a haber un mayor acceso al financiamiento de este tipo de infraestructuras energética, que permitió a las empresas desarrollar proyectos cada vez más grandes ${ }^{33}$. La caída de los costes de producción ha facilitado esta expansión de la energía eólica marina, que se ha acentuado en los últimos años. Mientras en 2015 el precio de la electricidad proveniente de la energía eólica marina era de \$150-200 USD/MWh, en 2019 se redujo a me-

\footnotetext{
${ }_{28}$ En este sentido SANZ señala que en la Comunicación sobre "El apoyo a la electricidad generada a partir de fuentes de energía renovable" se hace referencia "a los proyectos de energía eólica marina, si bien se ponen de manifiesto los obstáculos y las barreras que todavía existen en los Estados miembros". Lo mismo sucede en el Libro Verde "Estrategia para una energía sostenible, competitiva y segura", de 2006, en que se advierte de la necesidad de crear un marco político de apoyo para que se desarrolle todo el potencial de la energía eólica marina. SANZ 2007, 909-910.

29 Comisión Europea 2008, 1-2.

30 COMISIÓN EUROPEA 2010.

31 Participaron en esta Iniciativa Bélgica, Países Bajos, Luxemburgo, Alemania, Francia, Dinamarca, Suecia, Reino Unido, Irlanda y Noruega.

32 Comisión EUROPEA 2010.

33 European Wind Energy Association 2011, 10-17; Comisión Europea 2010.
} 
nos de $\$ 60$ USD/MWh ${ }^{34}$, destacando las subastas adjudicadas en Francia y Reino Unido, en las que se pagaron 44/MWh y 40,63 f/MWh, respectivamente ${ }^{35}$.

La inversión en eólica offshore aumentó desde los \$8.000 millones de dólares en 2010 a $\$ 20.000$ millones en 2018, representando casi una cuarta parte de la inversión mundial en el sector eólico y el 6\% de toda la inversión en renovables ${ }^{36}$. Durante 2020, en Europa se han invertido $€ 26.000$ millones de euros para financiar 7,1 MW de nuevos proyectos de eólica marina offshore que entrarán en funcionamiento en los próximos años ${ }^{37}$.

Asimismo, el desarrollo tecnológico de la industria continúa su progresión, aportando en la consolidación de la industria eólica offshore. En 2010, los fabricantes comienzan a ofrecer nuevos prototipos de turbinas marinas de 6 y $7 \mathrm{MW}$, que permitía a los nuevos proyectos aumentar exponencialmente la capacidad de los parques eólicos marinos. Hoy, los nuevos aerogeneradores marinos que poseen una potencia promedio de $10 \mathrm{MW}$, y los anuncios de prototipos de $14 \mathrm{MW}$, que estarán disponibles a partir de 2024, han favorecido el aumento de la capacidad de los nuevos parques eólicos marinos ${ }^{38}$.

En el mismo sentido, también ha habido avances en la tecnología referida a los soportes de los aerogeneradores. Junto a las estructuras rígidas de aerogeneradores que requieren de anclaje directo al suelo marino -turbinas eólicas fijas-, comienzan a desarrollarse estructuras flotantes, que permite instalar los parques en aguas cada vez más profundas y a distancias mayores desde la costa -energía eólica marina flotante-. Así, mientras las primeras infraestructuras eólicas marinas se instalaban a profundidades no superiores a 40 mts. de profundidad, y a unos pocos kilómetros de la costa, hoy existen parques a 100 metros de profundidad en Reino Unido y se están construyendo proyectos en países como Alemania, Reino Unido, Bélgica o Dinamarca, a más de $200 \mathrm{mts}$. del suelo marino, y a distancias superiores a $100 \mathrm{~km}$. de la costa, gracias a los aerogeneradores flotantes ${ }^{39}$.

Los parques eólicos se alejan cada vez más de la línea costera y comienzan a ubicarse en aguas profundas. Las turbinas eólicas marinas están alcanzando capacidades inimaginables hace solo unos pocos años atrás. Un claro ejemplo de los avances de la tecnología eólica marina podemos encontrarlo en el reciente anuncio del proyecto de parque eólico Sofía, que desarrollará una importante empresa alemana en las costas del Mar del Norte Británico; podrá producir 1400 MW de electricidad gracias a sus 100 aerogeneradores

\footnotetext{
34 Ocean Renewable Energy Action Coalition 2020, 16; Comisión Europea 2020-a, 4.

35 Ramírez; Fraile y Brindles 2020, 37.

36 Global Wind Energy Council 2020-a, 17.

37 Energías Renovables 2021.

38 Global Wind Energy Council GWEC 2020-a, 3-8; Comisión Europea 2020-a, 2-6.

39 Para revisar el fuerte desarrollo tecnológico que ha tenido la industria eólica marina, véase: Ramírez; Fraile y Brindles 2021, 15-20; Olivares 2021, 570-574; Comisión Europea 2020-b, 2-5.
} 
de 14 MW. Se instalará a $195 \mathrm{Km}$. de la costa en el norte de Inglaterra y entrará en funcionamiento en $2025^{40}$.

Como vemos, todos estos elementos, sumado al desarrollo de un marco jurídico favorable que incluye múltiples esquemas de incentivos -que serán revisados en el siguiente apartado-, han dado lugar a un crecimiento exponencial de esta fuente energética en los últimos diez años, situándola como "una de las tecnologías más dinámicas en el sistema energético"41. De esta forma, los $3 \mathrm{GW}$ de capacidad instalada en eólica marina en 2010, aumentaron a 11 GW en 2015, y para 2019 esa potencia se había casi triplicado para llegar a los 29,1 GW, que se distribuyen entre Europa, que concentra el $75,2 \%$ ese la capacidad instalada, y el resto del mundo con el 24,8\%, donde destacan China, Corea del Sur, Taiwan, Vietnam, Japón y Estados Unidos ${ }^{42}$.

En este escenario favorable, la Estrategia europea de 2020 para esta fuente energética, expresa que la eólica marina en Europa debe alcanzar los 300 GW en 2050 para cumplir con su objetivo de ser climáticamente neutra en dicho año ${ }^{43}$. A nivel global, se comparte ese optimismo. IEA estima que en el 2040 la capacidad eólica marina mundial se multiplicará por quince respecto de la capacidad instalada en 2018, llegando a los 560 GW, con un crecimiento importante en Asia y Estados Unidos, además de Europa ${ }^{44}$.

La actual fase de consolidación y expansión de la energía eólica marina está claramente vinculada a la que identificamos en los apartados anteriores como segunda fase del desarrollo de las energías renovables, en cuanto dimensión de la Acción por el Clima. En este sentido, la Comisión Europea reconoce el rol esencial que jugará la eólica offshore en el avance de una Economía neutra en emisiones, así como en el desarrollo de otros objetivos relacionados, como "aumentar el uso directo de la electricidad para un espectro más amplio de usos finales y apoyar la electrificación indirecta mediante el hidrógeno y los combustibles sintéticos" $" 45$.

Asimismo, el Pacto Verde europeo entrega a la energía eólica marina un papel decisivo en el cumplimiento de los objetivos de neutralidad climática del año 2050 y de reducción de emisiones de gases de efecto invernadero de $2030^{46}$. Además, la International Energy Agency señala que esta tecnología podría ahorrarnos entre cinco y siete millones de toneladas de las emisiones de $\mathrm{CO} 2$ del sector energético a nivel mundial ${ }^{47}$.

\footnotetext{
40 Puede revisarse la noticia en DIALEC 2021.

41 International Energy Agency 2019-a, 15.

42 Global Wind Energy Council 2020-a, 12.

43 Comisión Europea 2020-a, 8.

44 International Energy Agency 2019-a, 27-29.

45 Comisión Europea 2020-a, 1.

46 Comisión Europea 2019, 7.

47 International Energy Agency 2019-a, 3-14.
} 
Este rol principal de la eólica offshore en la estrategia global para el cambio climático es asumido también por los diversos países. Así, por ejemplo, Singh explica el interés de Reino Unido en esta fuente energética señalando que dicho Estado considera a la eólica offshore como una tecnología clave en sus planes a largo plazo para descarbonizar su generación de electricidad ${ }^{48}$.

Estamos, por tanto, ante una tecnología que se integra de manera natural en la Acción por el Clima y que facilita a los países la transición hacia un modelo energético poscarbono ${ }^{49}$. Su desarrollo está estrechamente relacionado con las diversas estrategias en la lucha contra el cambio climático. La energía eólica marina es parte de lo que Cassotta identifica como un nuevo modelo de inversión, centrado en la sostenibilidad, que permitirá a los países cumplir los objetivos de reducción de emisiones para limitar el calentamiento global a $2^{\circ} \mathrm{C}$ referidas en el Acuerdo de París ${ }^{50}$.

Sin embargo, a pesar de estas increíbles proyecciones de crecimiento de la energía eólica marina a nivel mundial y de su aporte en el avance hacia una Economía neutra en carbono, en América Latina no existen iniciativos relevantes para sumar a esta fuente energética en el combinado energético de la región. El Banco Mundial ha señalado recientemente que la capacidad técnica potencial del planeta es de 15.6 TW, de los cuales 6.3 TW se encuentran en la región de Latinoamérica y el Caribe ${ }^{51}$, y únicamente Brasil ha anunciado hace pocos meses un proyecto para desarrollar parques eólicos marinos en los Estados de Ceará y Rio Grande do Norte, aunque seguramente la intensidad de la crisis sanitaria que vive ese país con el Covid-19, afectará estos planes ${ }^{52}$.

Chile, que posee un potencial técnico para instalar 957 GW de energía eólica offshore -131 GW con aerogeneradores fijos y 826 GW de tecnología flotante ${ }^{53}-$, no escapa a esta realidad de la región. Aunque ha desplegado en la última década una activa política de desarrollo de energías renovables, que le ha permitido llegar en el año 2020 al 25\% de su capacidad instalada con energías eólica y solar ${ }^{54}$, no ha mostrado interés en implementar un plan de acción para la energía eólica marina.

Desde esta perspectiva, estimamos que la ausencia de incentivos a la industria eléctrica nacional para desarrollar proyectos eólicos marinos limitará en el mediano y largo plazo las posibilidades de Chile de lograr los objetivos de neutralidad climática en el horizonte 2050. Asimismo, las energías renovables consolidadas en el territorio nacional -la eólica terrestre y la solar fotovoltaica- hacen una utilización intensiva del territorio, que comenzará a gene-

\footnotetext{
48 SINGH 2017, 63.

49 Sobre esta idea, véase Olivares, 2019.

50 CASSOTTA 2016, 196.

51 World Bank \& Energy Sector Management Assistance Program 2020.

52 Revista Eólica y del Vehículo Eléctrico ReVE 2020.

53 World Bank \& Energy Sector Management Assistance Program 2020.

54 Energías Renovables 2020.
} 
rar tensiones con las comunidades locales por el uso del suelo. Finalmente, la necesidad de lograr una matriz baja en emisiones de carbono obliga a no desechar, a priori, ninguna tecnología de energías renovables existentes.

No obstante, las reducciones en los costes de producción que se han logrado, así como el progreso tecnológico conseguido para los aerogeneradores -tanto en su capacidad como en sus características (estructuras fijas y flotantes)-, permiten ser optimistas sobre el futuro de la energía eólica offshore en el país. Por ello, analizaremos a continuación las principales características que debe tener un régimen jurídico de promoción de esta fuente energética, y que podrían servir para el impulso de la eólica marina en las costas chilenas, siguiendo la senda que marca el panorama energético mundial.

\section{Marco jurídico para la eólica marina}

Como hemos señalado, los costes de la producción de electricidad con energía eólica marina se han ido reduciendo progresivamente hasta alcanzar en algunas subastas de países como Francia o Inglaterra unos precios cercanos a las de otras fuentes renovables consolidadas -eólica terrestre y solar ${ }^{55}$-. No obstante, todavía sus costes de producción siguen siendo mayores a estas últimas.

La tecnología en aerogeneradores y redes de transmisión marina ha ido venciendo los distintos obstáculos económicos y técnicos que limitaban las posibilidades de expansión de esta fuente energética, y han generado importantes avances en su competitividad ${ }^{56}$. Sin embargo, la instalación de parques eólicos en el mar constituye un importante desafío tecnológico para los estados, que no todos están dispuestos a asumir.

Asimismo, para países como Chile, con extensos territorios, resulta poco atractivo introducir una tecnología que todavía tiene costes superiores que otras alternativas ya maduras, y que además implican un importante reto técnico. Tienen todavía una gran cantidad de suelo disponible para desarrollar las energías renovables terrestres, que además son más baratas y técnicamente menos complejas. En cambio, resulta lógico que países como Dinamarca, Países Bajos o Bélgica, hayan comenzado a mirar al mar para encontrar soluciones a sus problemas de abastecimiento energético.

No obstante, como hemos advertido previamente, el tamaño cada vez mayor de los nuevos parques eólicos terrestres, solares fotovoltaicos y termosolares, está comenzando a generar problemas con las comunidades locales por el uso del suelo y ello obligará a los países costeros a buscar respuestas en los océanos ${ }^{57}$.

\footnotetext{
55 Lo mismo puede decirse de otras fuentes energéticas como la energía nuclear o las energías fósiles, pero en este trabajo limitaremos la comparación a las otras energías renovables más expandidas en el mundo.

56 COMISIÓN EUROPEA 2020-a, 22-24.

57 Solo a modo de ejemplo, recordaremos la sentencia Rol No 10.090-2011 de la Corte Suprema, que acogió un recurso de protección presentada por la comunidad en Chiloé en contra del proyecto "Parque Eólico Chiloé", obligándolo a someterse a un estudio de impacto ambiental.
} 
De la misma manera, la energía eólica marina responde a una nueva lógica energética, orientada por la estrategia global por el clima, y que exige a los Estados mayores compromisos en materia climática. Las decisiones de política energética no deben ser adoptadas por los países teniendo como único objetivo la reducción de costes la electricidad. Este debe conciliarse con otros dos objetivos igual de relevantes: la descarbonización de la matriz y fiabilidad del sistema. Y la energía eólica marina aporta especialmente en estos dos aspectos, al mismo tiempo que está reduciendo progresivamente sus costes de producción.

Por otra parte, la Acción por el Clima obliga a los países costeros a revisar su relación con los océanos en dos sentidos diversos, aunque estrechamente relacionados: reformular sus políticas de protección de los mares y los océanos, para adaptarlas a la Acción por el Clima, y; explorar las posibilidades de crecimiento sostenible que proporcionan los océanos, en lo que se ha venido a denominar Economía Azul. En este último punto, la energía marina es una de las más importantes formas de uso sostenible de los océanos para la adaptación al cambio climático y la reducción de emisiones de $\mathrm{CO} 2^{58}$.

Siguiendo a Levitt, las principales ventajas de la eólica offshore son las siguientes: i) el recurso de energía renovable más grande para muchos estados costeros; ii) la energía eólica marina ofrece beneficios económicos sustanciales y reducciones significativas en $\mathrm{CO} 2$ y otros contaminantes dañinos; iii) el coste privado de la energía eólica marina es menor que el de la mayoría de las otras nuevas tecnologías de energía renovables (aunque es más costoso que la eólica terrestre y la mayoría de las otras tecnologías de generación convencionales) ${ }^{59}$.

Asimismo, la energía eólica marina también presenta algunos beneficios respecto de la eólica terrestre: i) los vientos son más fuertes y constantes que en tierra, lo que permite una producción sensiblemente mayor por unidad instalada; ii) las turbinas eólicas pueden ser de mayor tamaño que en tierra, por las restricciones de transporte de grandes estructuras en tierra; iii) los parques eólicos marinos tienen menos posibilidades de causar problemas a las poblaciones próximas -ruido, objeciones estéticas, etc.-; iv) estas infraestructuras pueden resultar ventajosos para la protección de los ecosistemas marinos y producir sinergias con otros usos del entorno marino, como la acuicultura ${ }^{60}$.

Teniendo en cuenta estos méritos, diversos países como Francia, Reino Unido, Estados Unidos o China han anunciado ambiciosos planes para desa-

\footnotetext{
58 No profundizaremos en esta idea, por no ser parte de los objetivos de este estudio. Solo nos limitaremos a resaltar la importancia de que los países miren a los océanos como parte de su estrategia nacional para la adaptación al cambio climático. Sobre esta idea, véase: ComISIÓN EuROPEA 2014; COMISIÓN EUROPEA 2012.

59 LEVITT et al. 2011, 6408.

60 Singh 2017, 67; Comisión Europea 2008, 1-2.
} 
rrollar este recurso energético ${ }^{61}$. De esta forma, puede proyectarse que las expectativas de los distintos estudios que pronostican un fuerte crecimiento de la eólica marina, hasta consolidarse como una energía de referencia de la matriz energética en 2040-2050, no están siendo exageradas por un optimismo injustificado ${ }^{62}$.

Ante este panorama, revisaremos las principales características de los regímenes jurídicos que han apostado por el desarrollo de la energía eólica marina, y que pueden ser útiles para el desarrollo de un marco jurídico que estimule el desarrollo de esta tecnología en las costas nacionales.

\section{Una estrategia clara y un marco normativo integral}

El incentivo a la energía eólica marina debe comenzar por establecer un marco normativo que exprese una estrategia clara para esta fuente energética, acompañada de normas que se hagan cargo de sus características técnicas y económicas. Como hemos señalado, el desarrollo de la eólica marina conlleva una complejidad técnica que repercute en una alta inversión inicial. Como consecuencia, la instalación de parques eólicos en el mar y el despliegue de una red eléctrica marina que permita verter la electricidad en el sistema eléctrico debe iniciarse a partir de una estrategia pública de incentivo a este recurso energético y el impulso de un régimen jurídico que acompañe su desarrollo.

Sin embargo, este marco normativo no se debe limitar únicamente a establecer un conjunto de incentivos que hagan viable económicamente el desarrollo de este tipo de proyectos. Debe diseñarse un marco normativo más amplio, integral, que aborde las diversas cuestiones relativas a esta tecnología.

En primer lugar, se debe diseñar una estrategia para el impulso de esta energía, que contenga una referencia explícita a la oferta de energía eólica marina que se prevé en un periodo de tiempo concreto, otorgando confianza a los inversores ${ }^{63}$. Así, por ejemplo, el Plan anunciado por la Administración Biden, el 29 de marzo de 2021, estableció la ambiciosa meta de desplegar 30 GW de energía eólica marina para $2030^{64}$. Por su parte, Reino Unido ex-

${ }_{61}$ Entre estos planes, podemos destacar a Francia que en 2020 presentó su "Plan National Intégré Énergíe-Climat de la France" que proyectó para 2028 la instalación de 5,2 a 6,2 GW de eólica Marina (Gouvernement de France 2020). También en 2020, Reino Unido elaboró el documento "The Ten Point Plan for a Green Industrial Revolution" que propone aumentar la energía eólica marina desde los $10 \mathrm{GW}$ actuales a $40 \mathrm{GW}$ en 2030 (HM Government 2020). Por último, el 21 de marzo de 2021 Estados Unidos anunciaba que expandiría su capacidad eólica marina hasta los 30 GW para 2030, como parte de su plan de creación de empleo verde. Esta última medida puede verse en: https://www.whitehouse.gov/briefing-room/statements-releases/2021/03/29/ fact-sheet-biden-administration-jumpstarts-offshore-wind-energy-projects-to-create-jobs/

62 Sobre esta afirmación, recuerde los estudios antes referidos de: WorLD BANK \& ENERGY SeCtOR Management Assistance Program 2020; Commission Europea 2020-a; International Energy Agency 2019-a.

${ }^{63}$ Global Wind Energy Council 2020-b, 4.

64 Véase este Plan en: WH.GOV, disponible en: https://www.whitehouse.gov/briefing-room/ statements-releases/2021/03/29/fact-sheet-biden-administration-jumpstarts-offshore-wind-energy-projects-to-create-jobs. 
presa en el documento The Ten Point Plan for a Green Industrial Revolution que producirá $40 \mathrm{GW}$ de eólica offshore, también para 203065. Por último, Francia ha incluido la meta de aumentar en 5,2-6,2 GW su producción de electricidad con energía eólica marina para $2028^{66}$.

Cabe mencionar que en los tres casos mencionados, las acciones referidas al crecimiento de la energía eólica marina formaban parte de una estrategia climática más amplia, que contemplaba un conjunto de medidas para lograr la neutralidad climática en 2050. Asimismo, en todos ellos también se justificaba la acción para el desarrollo de las energías renovables en su positivo impacto en el empleo, que Estados Unidos ha cifrado en 44.000 nuevos puestos de trabajo asociados directamente a los nuevos proyectos eólicos marinos y 33.000 empleos adicionales en actividades relacionadas con esta industria, para 2030. Del mismo modo, Reino Unido ha estimado que la industria eólica marina generará hasta 60.000 nuevos puestos de trabajo en $2030^{67}$.

Otras cuestiones que deben ser abordadas por un marco normativo que facilite la implementación de proyectos marinos dicen relación con aspectos como la propiedad del fondo marino; el sistema de concesiones para su uso, así como la ampliación del proyecto; el proceso de servidumbres para desplegar la red de transporte marino; o definiciones sobre qué hacer con las instalaciones marinas cuando expiren las autorizaciones iniciales ${ }^{68}$.

Finalmente, la seguridad jurídica también es necesaria para implementar este tipo de actividades, que requieren de plazos extensos para entrar en funcionamiento y también para el retorno de la inversión ${ }^{69}$. En este último sentido, por ejemplo, en Alemania la Erneuerbare-Energien-Gesetz de 2016 (EEG-2016) estableció un régimen de incentivos para la eólica marina, con un período de soporte de 20 años, que podía ser aumentado si la localización de estos parques era a mayor distancia de la costa o profundidad ${ }^{70}$. Además, todo el marco jurídico relativo a las energías renovables en general está orientadas por el principio de prioridad de estas fuentes energéticas, otorgando un alto grado de confiabilidad en la planificación por parte de los inversores, facilitando el desarrollo de proyectos con un alto coste como los parques eólicos marinos ${ }^{71}$.

En definitiva, de manera general, para incentivar el desarrollo de parques eólicos marinos debe pensarse en un marco jurídico de largo aliento,

\footnotetext{
65 HM Government 2020, 8.

66 Gouvernement de France 2020, 65.

67 HM Government 2020, 9.

68 SINGH 2017, 66.

69 GWEC estima que la entrada en funcionamiento de un parque eólico marino tarda unos 8 años desde que se diseña el proyecto. Asimismo, el periodo de retorno de la inversión no es inferior a 20 o 30 años, según la entidad del proyecto. Global Wind Energy Council 2020-b.

70 Schomerus, Maly 2017, 38.

71 Blomberg 2014, 46.
} 
sistémico, coherente y funcional, que permita proyectar un crecimiento de esta fuente energética, al alero de una política climática nacional ${ }^{72}$.

\section{La planificación del espacio marítimo}

Una segunda cuestión, esencial para generar un entorno normativo favorable a la implementación de la energía eólica offshore, es la existencia de una planificación espacial marina, que incluya las áreas en que se puedan desarrollar los parques eólicos marinos ${ }^{73}$. Asimismo, la Comisión Europea ha señalado que para alcanzar la meta de contar con una capacidad instalada en la UE de 300 GW de energía eólica marina para 2050, se requiere identificar y usar un gran número de emplazamientos para la producción de energía renovable marina y la conexión a la red de transporte de energía. Este ordenamiento del espacio marítimo permite a las autoridades públicas planificar esta evolución a largo plazo en una fase temprana, y conciliar esta actividad energética con otras que se puedan realizar, como el transporte marítimo, la protección de los ecosistemas marinos o la pesca ${ }^{74}$.

Una importante problemática que se resuelve con la ordenación del espacio marítimo es la convivencia de los parques eólicos marinos con áreas de protección de los ecosistemas marinos. La Unión Europea señala expresamente que:

los espacios marítimos designados para la explotación marina deben ser compatibles con la protección de la biodiversidad, tener en cuenta las consecuencias socioeconómicas para los sectores que dependan de la buena salud de los ecosistemas marinos e integrar en la medida de los posible otros usos del mar $^{75}$.

Una buena forma de abordar la convivencia entre las áreas de desarrollo eólico marino y los espacios protegidos es el modelo alemán que establece zonas de exclusión, prohibiendo la instalación de los parques eólicos offshore a menos de $40 \mathrm{~km}$. de distancia de las áreas de protección marina ${ }^{76}$.

Las distintas estrategias de los países que han desarrollado están orientadas a la identificación previa de las áreas en que es factible instalar pro-

\footnotetext{
72 Especialmente, debe pensarse en un nuevo sistema autorizatorio del espacio marítimo, que facilite el despliegue de los parques eólicos marinos (u otras, como las plantas desalinizadoras), en Chile. Mientras no haya una reforma integral del régimen de autorizaciones de uso de la zona marítima, que facilite la autorización tipo de infraestructuras marinas, el modelo vigente será una barrera a su implantación. Este relevante tema será abordado en una nueva investigación que está en curso.

73 Comisión Europea 2014, 7. En este sentido, por ejemplo, en Noruega la Norwegian Water Resources and Energy Directorate (NVE), en 2017 había identificado 15 áreas para instalar parques eólicos marinos. BANET 2017-a, 148. También en Francia, la Stratégie Nationale pour la Mer et le Littoral, SNML, de 2017, ha identificado 100 zonas en que podían instalarse estas infraestructuras. BANET 2017-b, 96.

74 COMISIÓN EUROPEA 2020-a, 8.

75 ldem.

76 Schomerus, Maly 2017, 33.
} 
yectos eólicos marinos, como una forma de reducir costes a las empresas, facilitar el proceso de autorización administrativa, conciliar la explotación energética de los océanos con la protección de sus ecosistemas, y prevenir en la medida de lo posible conflictos con la comunidad. Así por ejemplo, en Noruega, la Norwegian Water Resources and Energy Directorate (NVE), en 2017 había identificado 15 áreas para instalar parques eólicos marinos, que posteriormente han sido licitados en procesos competitivos ${ }^{77}$.

Lo mismo ha hecho Japón, que en noviembre de 2018 presentó ofertas competitivas para zonas de promoción identificadas con antelación, en un nuevo marco nacional de incentivo a proyectos eólicos marinos. Además, de acuerdo con la normativa japonesa, el precio es solo uno de los elementos de competencias para los promotores en estos procesos de licitación, al cual debe sumarse a la idoneidad de los planes de ocupación en las zonas de promoción ${ }^{78}$.

Como vemos, el desarrollo de la energía eólica marina ha sido parte de una planificación del espacio marítimo por los países que le permite conciliar los distintos usos de los océanos. Esta ordenación marina se representa en la identificación previa por parte del Estado de las diversas zonas en que es factible ambiental y técnicamente la instalación de parques eólicos marinos, para posteriormente realizar su adjudicación mediante procesos licitatorios, donde el precio es solo uno de los factores evaluados, que incluyen además otros aspectos referidos a la calidad del proyecto y su relación con el entorno.

\section{Un régimen eficiente de permisos administrativos}

Uno de los temas más complejos para el desarrollo de proyectos eólicos marinos ha sido el régimen de permisos administrativos y ambientales para su construcción y funcionamiento. Cassotta ha señalado que la falta de desarrollo de la energía eólica marina en Italia tiene como principal causa el engorroso sistema de licencias y autorizaciones del país, que ha operado como una verdadera barrera para el sector ${ }^{79}$.

En Inglaterra y Gales, para proyectos de más de 100 MW han implementado una ventanilla única gestionada por un organismo público denominado"Planning Inspectorate", que concede las autorizaciones ambientales en tierra y en alta mar y también las autorizaciones de generación eléctrica. La solicitud de un proyecto eólico marino debe ser presentada a la Planning Inspectora quien la examina y tramita, debiendo presentar un reporte ante la Secretaría de Estado respectiva, incluida la recomendación de aprobación o rechazo, en un plazo de seis meses.

Para los proyectos offshore de más de 100 MW, será la Secretary of State for Communities and Local Government, quien emitirá en un plazo de 3

\footnotetext{
77 BANET 2017-a, 148-149.

78 Global Wind Energy Council 2020-a, 34.

79 Cassotta 2017, 91.
} 
meses una "development consent order", en caso de acogerse el proyecto. En cambio, los proyectos de menos de $100 \mathrm{MW}$ solo necesitan una "required pursuant" otorgada por la "Marine Management Organisation (MMO)"80.

Alemania también ha implementado un modelo de ventanilla única que incluye a una autoridad que asume la gestión del procedimiento de licencia -Bundesamt for Sddschifffahrt und Hydrografie BSH-. Esta se encarga de informar del proyecto y requerir las opiniones de los otros órganos involucrados en la autorización del proyecto, incluidas las organizaciones de protección ambiental. Finalmente, la BSH toma la decisión de otorgamiento o no de la licencia de explotación, tomando en consideración todas las opiniones recabadas ${ }^{81}$.

Debemos tener en consideración que en un procedimiento de autorización para la entrada en funcionamiento de un parque eólico marino participan una serie de organismos sectoriales, con competencias en el espacio marítimo, medioambiental, energético, etc. Asimismo, se involucran órganos de carácter nacional como regional y local. De esta forma, es fundamental adoptar un sistema de ventanilla única, que incluya la designación de un ente concreto que asuma la gestión de este proyecto, coordinando las diversas opiniones sobre el proyecto y que presente la propuesta fundada de aprobación o rechazo al órgano que finalmente decide.

\section{El desarrollo de redes marinas de transmisión}

Uno de los puntos más complejos de la energía eólica marina es el desarrollo de una red marina de transmisión que permita verter la electricidad en el sistema eléctrico del país. Hemos mencionado antes, que la Unión Europea puso énfasis en la necesidad de implementar una red marina interconectada en el Mar del Norte, como parte de la estrategia de promoción de esta fuente energética. En el mismo sentido, la recientemente anunciada estrategia de Reino Unido para acelerar su transición energética, incluye en el plan de acción relativo a la energía eólica marina una revisión del plan de desarrollo de la red eléctrica marina ${ }^{82}$.

La Comisión Europea ha advertido que "la mayoría de los parques eólicos marinos existentes se han desplegado como proyectos nacionales conectados directamente a la costa a través de enlaces radiales". Que este modelo es útil para comenzar a implantar la energía eólica marina, pero que esta modalidad debe ser complementada con el desarrollo de "interconectores transfronterizos para el comercio de electricidad y la seguridad de suministro" 83 .

Un sistema novedoso es el utilizado por Inglaterra y Gales, donde los desarrolladores de un proyecto eólico marino deben solicitar por separado

\footnotetext{
80 Singh 2017, 69-80; Global Wind Energy Council 2014, 39.

81 Schomerus, Maly 2017, 33-38.

82 HM GovernMENT 2020, 9.

83 Comisión Europea 2020-a, 12.
} 
al operador del sistema de transmisión -Office of Gas and Electricity Markets (OFGEN)- la autorización de conexión a la red. La forma que se ha usado hasta ahora para la implementación de las redes de transmisión marina es que los generadores desarrollan y construyen estas infraestructuras y luego deben venderlas, en un plazo de 18 meses, a través de una licitación competitiva, a uno de los propietarios de transmisión marina (Offshore transmission owner OFTO), que adquieren y operan la red marina a través de tarifas de transmisión ${ }^{84}$.

Debemos reconocer que este es un punto crítico en la implementación de un sistema de parques eólicos en Chile. El sistema de redes de interconexión del Mar del Norte sirve como referencia a los demás países sobre cómo avanzar en el mallado sostenible de una red de transmisión marina, pero esa realidad, con diversos países participando en el desarrollo de esta red que permite además la interconexión energética de estos países, difiere mucho de las características de la costa chilena, extensa y sin vecinos con quienes compartir esta red, a excepción del extremo norte con Perú.

Una solución que plantea la Unión Europea y que podría ser útil a Chile, es el desarrollo de islas energéticas, proyectos híbridos donde se instalan distintos parques eólicos marinos alrededor de una isla artificial que puede albergar un sistema de almacenamiento de electricidad, que se conecta al sistema eléctrico nacional a través de una única red. Además, pueden también operar en estas islas instalaciones de hidrógeno verde, que reduciría aún más los costes ${ }^{85}$. En febrero de 2021 Dinamarca ha anunciado la construcción de la primera isla energética del mundo, que estará conectada a los parques circundantes y suministrará electricidad a los países de su entorno ${ }^{86}$.

\section{Un adecuado esquema de incentivos}

Finalmente, revisaremos los distintos esquemas de incentivos regulatorios utilizados por los países para el desarrollo de la energía eólica marina. En el Reino Unido, la Energy Bill 2015/16, de 2015, introdujo un nuevo sistema de incentivos para proyectos de generación eléctrica bajos en emisiones de carbono, denominado contratos por diferencia (CfD). En este esquema, la empresa generadora suscribe un CfD con la empresa pública Low Carbon Contracts Company (LCCC) ${ }^{87}$, que es un contrato de largo plazo en el que se fija un precio fijo por la electricidad producida, que refleja el costo de invertir en una tecnología bajo en emisiones de carbono: el precio de ejercicio (strike price). En virtud del CfD, la empresa eléctrica vende la electricidad en el mercado mayorista a precio de mercado, y cuando este es inferior al precio de ejercicio, recibe un pago adicional por parte de la LCCC, equivalente a la diferencia entre ambos precios. Como contrapartida, cuando el precio de

\footnotetext{
84 Office of Gas and Electricity Markets 2014; Global Wind Energy Council 2014, 39.

85 Comisión Europea 2020-a, 13.

86 LÓPEZ 2021.

87 El sistema de CfD es financiado con gravámenes y cargas impuestas a las empresas eléctricas que operan en Reino Unido.
} 
mercado es mayor al precio de ejercicio, la empresa eléctrica debe pagar la diferencia de precio a la LCCC ${ }^{88}$.

En cambio, Alemania con la Erneuerbare-Energien-Gesetz de 2014 (EEG-2014) estableció un sistema de incentivos feed-in-premium. Es decir, una prima sobre el precio de mercado de la electricidad. Este régimen tenía dos modelos: uno básico (establecido en la sección 50, EEG-2014) que garantizaba una prima de 15.40 céntimos de euro por kWh para un periodo de veinte años, que podía ser extendido 0,5 mes por cada milla náutica que el parque eólico marino se alejaba de la costa, a partir de las 12 millas náuticas marinas, y 1,7 meses por cada metro de profundidad del agua, iniciando desde los $30 \mathrm{mts}$. de profundidad. El segundo esquema, denominado modelo de aceleración (sección 50, subsección 2ª , EEG-2014), permitía al parque eólico recibir 19,4 céntimos de euro por kWh, durante los ocho primeros años si la instalación se ponía en marcha antes del 1 de enero de 2020, y para los siguientes años hasta completar su periodo de incentivos volvía al precio del modelo ordinario $(15,40 \text { céntimos de euro/kWh })^{89}$.

Este esquema de incentivos está siendo reemplazado por el gobierno alemán para los proyectos que se presenten a partir de 2021. El gobierno ha estimado que la tecnología eólica marina en las costas alemanas se encuentra lo suficientemente madura para poder competir en el mercado de electricidad a través de un sistema especializado de subastas. Para ello, ha adoptado el modelo danés, que entrará en funcionamiento plenamente en 2026, donde el gobierno subastará los sitios donde se pueden instalar los parques eólicos. Estos lugares contarán con los estudios de factibilidad previos pertinentes por parte de la Administración, incluido el acoplamiento con las conexiones de la red ${ }^{90}$.

Japón ha implantado en 2012 un régimen de feed-in-tariff para todas sus energías renovables, aunque con tarifas diferenciadas por tecnología. De esta forma, el Ministerio de Economía, Comercio e Industria aprobó en 2014 una tarifa para estas fuentes energéticas de 36 yenes/kWh $(0.28 € / \mathrm{kWh})$ para la eólica offshore, que se ha actualizado periódicamente ${ }^{91}$.

Noruega, en cambio, no tiene un régimen específico para la energía eólica marina. Los proyectos con esta tecnología pueden recurrir a los incentivos comunes para todas las energías renovables elegibles: el esquema de certificados verdes, o el sistema de soporte para la investigación y desarrollo de nuevas tecnologías (R\&D Policy). Por tanto, el modelo noruego está inmerso en un sistema general de incentivos al desarrollo tecnológico-climático

\footnotetext{
88 SINGH 2017, 69-73.

89 Schomerus, Maly 2017, 38-39; Blomberg 2014, 46-47.

90 Schomerus, Maly 2017, 39-40.

91 Global Wind Energy Council 2020-a, 58.
} 
que permita consolidar industrias enfocadas en la exportación de tecnologías hipocarbónicas ${ }^{92}$.

En cuanto al esquema de certificados verdes, general para todas las energías renovables elegibles (hidroelectricidad, eólica, solar, energía de los océanos, geotérmica y bioenergía), debe indicarse que se ha creado un mercado común de certificados verdes para Noruega y Suecia.

De acuerdo con la Electricity Certificates Act, N³9-2011, las empresas generadoras que utilizan tecnología renovable elegible reciben un certificado (denominado elcertificate) por cada MWh producido, para un período de 15 años. Estos elcertificates son ofrecidos en este mercado común sueco-noruego de certificados verdes. La demanda se estimula generando una obligación a los proveedores de electricidad y a los grandes consumidores de electricidad de entregar certificados equivalentes a una proporción de la electricidad que suministran o consumen, fijada por el regulador energético. Si estos no poseen los certificados respectivos, deben adquirirlos en este mercado o de forma bilateral. La autoridad establece la obligación de cuota que debe certificar cada empresa eléctrica o gran consumidor, pero el precio de los elcertificates los fija el mercado. Las cuotas establecidas por el regulador han ido subiendo progresivamente hasta 2020, para luego comenzar a reducirse nuevamente hasta 2035. En 2036 este programa dejará de funcionar ${ }^{93}$.

En Estados Unidos, a nivel federal, opera un sistema de financiamiento de energías renovables, incluida la eólica marina basado en el crédito fiscal a la producción (production tax credit, PTC) y, más recientemente, como alternativa se está usando el crédito fiscal a la inversión (Investment Max Credit, ITC). Mientras el sistema PTC es un crédito definido por los kWh de producción del parque de generación, en el ITC el financiamiento fiscal está delimitado por los gastos de capital. De esta forma, para los proyectos más intensivos en capital -como es el caso de los parques eólicos marinos-, les resulta más beneficioso este último sistema.

El reciente paquete de medidas anunciado por el Presidente Biden para incentivar la industria eólica offshore, fortalece este esquema de incentivos centrado en el crédito fiscal. Entre otras acciones ha enunciado el acceso a \$3 mil millones en capital de deuda para respaldar la industria eólica marina, a través del Departamento of Energy DOE. Como complemento se destinará un presupuesto de $\$ 230$ millones de dólares en préstamos para el desarrollo de infraestructura portuaria de apoyo a la eólica marina ${ }^{94}$.

Corea del Sur, en cambio, ha implementado a partir de 2010 un esquema de incentivos Renewable Energy Portfolio Standard (RPS) para incentivar las energías renovables en general. Por tanto, se establecieron metas de producción con energías renovables, que los operadores eléctricos debían cum-

\footnotetext{
92 BANET 2017-a, 142-143.

93 Banet 2017-a, 154-156; Energy Facts Norway 2019.

94 Schomerus, Maly 2017, 38-39; Blomberg 2014, 46-47.
} 
plir. Si no cumplen con las cuotas establecidas por ley, reciben fuertes sanciones económicas. Sin embargo, pueden suplir sus déficits en producción con renovables comprando certificados de suministro de energía renovable (REC). Este modelo tradicional de RPS, no obstante, tiene una particularidad: se ha creado una tabla multiplicadora de los REC para cada fuente energética renovable. De esta manera, se reconoce el mayor coste de unas energías renovables sobre otras y se incentiva ciertas tecnologías estratégicas como la eólica marina ${ }^{95}$.

Así, la tabla multiplicadora establece para la energía solar un factor de entre 0,7 y 1,5 por cada certificado de suministro de energía renovable que se adquiera; para la hidroelectricidad, la bio-energía y la eólica terrestre es de 1.0, y; para la eólica offshore el factor es de 1,5 si está a menos de $5 \mathrm{~km}$ de la costa, y si el parque eólico marino se ubica a más de $5 \mathrm{~km}$, será de 2,0 para el caso de las instalaciones finjas y de hasta 2,5 para tecnologías flotantes ${ }^{96}$.

En definitiva, los diversos esquemas de incentivos establecidos por los países para el incentivo de la energía eólica marina forman parte del régimen común de estímulos a todas las energías renovables, aunque reconocen las especialidades de dicha fuente energética, emanadas de sus complejidades técnicas y mayor coste, para establecer algunas modalidades que premian la inversión en esta tecnología.

\section{Conclusiones}

La electricidad es la fuente energética del futuro. Es una energía que puede lograr la neutralidad carbónica si es producida con energías limpias. De esta forma, las energías renovables han pasado a ser el centro neurálgico de la estrategia de descarbonización de la Economía mundial.

En este contexto, las posibilidades de la energía eólica marina, con una capacidad técnica de 15,6TW -según estimaciones del banco Mundial-, la convierten en una fuente energética esencial para la transición hacia un modelo energético climáticamente sostenible. Como hemos podido ver, el incentivo a este recurso energético forma parte de la estrategia que los países han comenzado a elaborar para la transición hacia la neutralidad climática.

Este rol que la comunidad internacional ha asignado a esta fuente energética, como parte de la acción global por el clima, sumado a la reducción de precios y el desarrollo técnico logrado, ha dado lugar a un importante avance de esta tecnología en los últimos 10 años. De esta forma, las proyecciones de organismos como la IEA o la UE sitúan a la eólica marina como una de las principales fuentes energéticas en el horizonte 2050.

En este sentido, si bien la Unión Europea reconoce que los costes de financiamiento representan entre el 35 y el $50 \%$ del costo total de generación

\footnotetext{
95 LeE 2017, 262-263.

96 íbíd., 263-264.
} 
con eólica marina, también recuerda que los distintos marcos y políticas de apoyo permiten ahora que los proyectos aseguren financiamiento de bajo coste en Europa, con licitaciones sin subsidio ${ }^{97}$. De esta forma, se espera que en los próximos años sea una fuente energética plenamente competitiva.

Como consecuencia, diversos países han comenzado a actualizar sus estrategias climáticas incorporando compromisos concretos en la capacidad instalada de eólica offshore para los próximos años. La transición hacia una Economía neutra en carbono exige incorporar todas las energías renovables disponibles para lograr una matriz energética libre de emisiones.

Al mismo tiempo, la implantación de la energía eólica marina permite estimular la innovación y el desarrollo tecnológico asociado a esta industria. Ello significa también un fuerte impulso al empleo cualificado, tal como recogen las propuestas presentadas recientemente por Reino Unido, Francia y Estados Unidos, respectivamente.

El desarrollo de la energía eólica marina requiere de un acompañamiento normativo que incluye una estrategia clara y régimen jurídico estable, funcional y sistémico, que otorgue seguridad jurídica a los inversores, por tratarse de una industria intensiva en inversión. Este marco debe incluir definiciones en torno al sistema de concesiones del suelo marino, su renovación o el desarrollo de la red marina de transmisión.

Asimismo, debe garantizarse un régimen de licencias y autorizaciones administrativas de ventanilla única, que evite los retrasos innecesarios en la tramitación de permisos, elevando los costes. La gestión de estas autorizaciones debe ser encargada a un único órgano especializado, quien se encargará de la tramitación, que incluye recabar la opinión de los diversos entes sectoriales competentes. La decisión final, podrá ser entregada a un órgano distinto que resuelve tomando en consideración todos los aspectos relacionados con la tramitación del proyecto, aceptando o rechazando la propuesta entregada por el ente que ha tramitado la solicitud.

En cuanto al desarrollo de las redes marinas para transportar la electricidad al sistema eléctrico nacional, se trata, sin duda, de una de las cuestiones más complejas para la energía eólica marina. Nos parece que la mejor solución es el impulso de islas energéticas que agrupen un conjunto de parques eólicos marinos que se conecten a través de una única red con el territorio nacional.

Finalmente, los diversos esquemas de incentivos revisados nos permiten entender la importancia de la energía eólica marina en el futuro energético de los países. Si bien, no se han elaborado mecanismos de estímulos específicos para esta tecnología, sí se han realizado ajustes para incorporar sus características técnicas y económicas, atendida su relevancia en la transición hacia un modelo energético poscarbono.

97 International Renewable Energy Agency 2019-b, 12. 
Este debería ser el elemento central de una futura estrategia de promoción de esta tecnología en el país. Avanzar hacia la segunda fase de impulso de las energías renovables, y en particular de la eólica marina, donde el precio de la electricidad sea solo un elemento de decisión, que comparte importancia con la fiabilidad del sistema y la descarbonización de la matriz.

\section{Bibliografía citada}

Ariño, G. (Dir.) (2006). Energía en España y desafío europeo. Seguridad, concentración empresarial, competencia, regulación. Comares.

BANET, C. (2017-a). Legal Framework to Develop Offshore Wind Power in Norway. En A. Ming-Zhi y C. Fan. (Eds.), The development of a Comprehensive Legal Framework for the Promotion of Offshore Wind Power. The Lessons from Europe and Pacific Asia (pp. 141-185). Wolters Kluwer.

BAnet, C. (2017-b). Legal Framework to Develop Offshore Wind Power in France. En A. Ming-Zhi y C. Fan. (Ed.), The development of a Comprehensive Legal Framework for the of Offshore Wind Power. The Lessons from Europe and Pacific Asia (pp. 90-123). Wolters Kluwer.

Bertoldi, P. (Ed.) (2018). Guidebook 'How to develop a Sustainable Energy and Climate Action Plan (SECAP)'. Publications Office of the European Union.

BLOMBERG, S. (2014). The realization of offshore wind park projects in Germany - political environment, legal framework and bankability implications. Anchor Academic Publishing.

Bloomberg Nef (9 de noviembre de 2020). Climatescope Emerging Markets Outlook 2020. Energy transition in the world's fastest growing economies. Global Climatescope. https://global-climatescope.org/assets/data/reports/climatescope-2020-report-en.pdf.

Cassotta, S. (2017). Legal Framework to Develop Offshore Wind Power in Italy. En A. MingZ HI y C. FAN (Eds.). The development of a Comprehensive Legal Framework for the Promotion of Offshore Wind Power. The Lessons from Europe and Pacific Asia (pp. 87-102). Wolters Kluwer.

CassottA, S. (2016). The Paris Agreement in Logic of Multi-Regulatory Governance: A Step Forward to a New Concept of 'Global Progressive Adaptive-Mitigation'? Energy Environmental Law and Policy Series, 25 (6), 196-212.

DiALEc (31 de marzo de 2021). El parc eòlic mare Sofía, que RWE desenvolupa en aigües de la Mar del Nord britànica, serà el primer en equipar-se amb els aerogeneradors de 14 MW de Siemens-Gamesa. https://dialec.blogspot.com/2021/03/el-parc-eolic-marisofia-que-rwe.html?m=1.

DíAZ, V. (2016). Los retos de la energía eólica marina en España: El papel de las C.C.A.A. y la ordenación de los espacios marinos ante la Directiva 2014/89/CE. Actualidad Jurídica Ambiental, 56, 1-26.

Energías Renovables (8 de febrero de 2021). Europa invierte la cifra récord de 26.000 millones de euros en eólica marina. https://www.energias-renovables.com/eolica/europa-invierte-la-cifra-record-de-26-20210208.

Energías Renovables (29 de julio de 2020). La capacidad instalada renovable llega al $24 \%$ y genera más del 18\% del total. https://www.energias-renovables.com/panorama/la-capacidad-instalada-renovable-llega-al-24-20200729.

ENERGY FACTS NORWAY (2019). "Electricity certificates". https://energifaktanorge.no/en/regulation-of-the-energy-sector/elsertifikater/

EUROPEAN WIND ENERGY AsSOCIATION (2011). The European offshore wind industry key trends and statics 2010. http://www.ewea.org/fileadmin/files/library/publications/statistics/20110121_Offshore_stats_Full_Doc_final.pdf.

Global Wind Energy Council GWEC (2020a). Global Offshore Wind Report 2020. GWEC.

Global Wind Energy Council GWEC (2020b). Offshore Wind Market Readiness Assessment. GWEC. 
Global Wind Energy Council GWEC (2014). Offshore Wind Policy and Market Assessment. A Global Outlook. https://gwec.net/wp-content/uploads/2021/01/GWEC_Offshore-WindPolicy-and-Market-Assessment---A-global-outlook_2014.pdf.

Gouvernement de France (2020). Plan National Integre Energie-Climat de la France. https:// ec.europa.eu/energy/sites/ener/files/documents/fr_final_necp_main_fr.pdf.

Grupo Intergubernamental de Expertos sobre el Cambio Climático (2015). Cambio Climático 2014. Informe de síntesis. Contribuciones de los grupos de trabajo I, II, y III al Quinto Informe de Evaluación del Grupo Intergubernamental de Expertos sobre el Cambio Climático. IPCC.

HendeRson (2002). Offshore wind in Europe: The current state of the art. Refocus, 3 (2), 14-17.

HM Government (2020). The Ten Point Plan for a Green Industrial Revolution. https://assets. publishing.service.gov.uk/government/uploads/system/uploads/attachment_data/ file/936567/10_POINT_PLAN_BOOKLET.pdf.

International Renewable Energy Agency (2019a). Renewable capacity statistics 2019. IRENA.

International Renewable Energy Agency (2019b). Offshore Wind Outlook 2019. World Energy Outlook Special Report. https://webstore.iea.org/offshore-wind-outlook-2019-worldenergy-outlook-special-report.

International Energy Agency (2019a). Offshore Wind Outlook 2019. World Energy Outlook Special Report. IEA Publications.

International Energy Agency (2019b). Global Energy \& CO2 Status Report. The latest trends in energy and emissions in 2018. IEA Publications.

International Energy Agency (2019c). Renewables 2019. Analysis and forecast to 2024. IEA Publications.

KoOHI-FAYEGH, S. y Rosen, M. (2020). A review of energy storage types, applications and recent developments. Journal of Energy Storage, 27, 1-23.

LeE, E. (2017). Legal Framework to Develop Offshore Wind Power in Korea. En A. MING-ZHI y C. FAN (Eds.), The development of a Comprehensive Legal Framework for the Promotion of Offshore Wind Power. The Lessons from Europe and Pacific Asia (pp. 255-275). Wolters Kluwer.

LEVITT, A. et al. Pricing offshore wind power. Energy Policy, 39, pp. 6408-6421.

LÓPEZ, S. (19 de febrero de 2021). Dinamarca construirá la primera isla energética del mundo en el Mar del Norte. https://ielektro.es/2021/02/19/dinamarca-isla-energetica-eolica/.

LUCAS, A. (2017). Novedades del Sistema de Protección Internacional de Cambio Climático: el Acuerdo de París. Estudios Internacionales, 49 (186), 137-167.

Ocean Renewable Energy Action Coalition (2020). The power of our Ocean. https://gwec. net/oreac/.

Office of Gas and Electricity Markets (2014). Offshore Transmission (OFTO) regime, now fully in place, set to achieve further savings. https://www.ofgem.gov.uk/press-releases/ offshore-transmission-ofto-regime-now-fully-place-set-achieve-further-savings.

Olivares, A. (2021). Energía eólica marina en España, una asignatura pendiente. En L. Moreno y C. Villanueva (coords.), Anuario Iberoamericano de Derecho de la Energía. Regulación de la transición Energética. El derecho de la energía sostenible (vol. III) (pp. 165-193). Universidad Externado de Colombia.

Olivares, A. (2019). El papel que desempeña la tecnología en la construcción del nuevo modelo energético postcarbono. En L. Moreno y C. Villanueva (coords.), Anuario Iberoamericano de Derecho de la Energía. Regulación de la transición Energética (vol. II) (pp. 563-586), Universidad Externado de Colombia.

PAReJo, L. (2010). El sistema energético. En L. Parejo et al., Lecciones de Derecho Administrativo. Orden económico y sectores de referencia (pp. 597-644). Tirant lo Blanch.

Parlamento EuRopeo (2018). Emisiones de gases de efecto invernadero por país y sector (infografía). https://www.europarl.europa.eu/news/es/headlines/society/20180301STO98928/emisiones-de-gases-de-efecto-invernadero-por-pais-y-sector-infografia.

POLLITT, M. (1999). Issues in electricity market integration and liberalization. En L. BERGMAN et al., A European Market for Electricity? (pp. 27-86). Centre for Economic Policy Research. 
Programa de naciones las Naciones Unidas para el Desarrollo (2020). Objetivos de DesarroIlo Sostenible. https://www.undp.org/content/undp/es/home/sustainable-development-goals.html.

Ramírez, L.; Fraile, D. y Brindles, G. (2021). Offshore Wind in Europe. Key trends and Stistics 2020. WindEurope Report. https://windeurope.org/intelligence-platform/product/offshore-wind-in-europe-key-trends-and-statistics-2020/.

Ramírez, L., Fraile, D. y Brindles, G. (2020). Offshore Wind in Europe. Key trends and Stistics 2019. WindEurope Report. https://windeurope.org/wp-content/uploads/files/aboutwind/statistics/WindEurope-Annual-Offshore-Statistics-2019.pdf.

Revista Eolica y del Vehículo Eléctrico ReVE (7 de marzo de 2020). Primeros pasos de la eólica marina en Brasil. https://www.evwind.com/2020/03/07/primeros-pasos-de-la-eolica-marina-en-brasil/.

Rivero, P. (2011). Mi visión del sector eléctrico: ¿mercado o regulación? Cuadernos de Energía, 31 (2), 64-68.

Rodríguez, L.; Pereira, I. y Toval, N. (2011). Impactos ambientales de un parque eólico marino. Retos y oportunidades. El caso de Huelva. [Tesis de fin de Máster, Escuela de Organización Industrial].

SANZ, J. (2007). Aproximación al régimen jurídico sobre parques eólicos: Una asignatura pendiente. Anuario da Facultad de Dereito da Universidade da Coruña, 11, 899-924.0.

Schomerus, T. y Maly, C. (2017). Legal Framework to Develop Offshore Wind Power in Germany. En A. Ming-Zhi y C Fan (Eds.), The development of a Comprehensive Legal Framework for the Promotion of Offshore Wind Power. The Lessons from Europe and Pacific Asia (pp. 30-67). Wolters Kluwer.

SingH, N. (2017). Legal Framework to Develop Offshore Wind Power in United Kingdom. En A. Ming-Zhi y C. Fan (Ed.), The development of a Comprehensive Legal Framework for the Promotion of Offshore Wind Power. The Lessons from Europe and Pacific Asia (pp. 63-88). Wolters Kluwer.

WERNER, S. (2017). International review of district heating and cooling. Energy, 137, 617-631.

WIND EUROPE (2019). Offshore Wind in Europe. Key trends and statics 2018. https://windeurope.org/wp-content/uploads/files/about-wind/statistics/WindEurope-Annual-Offshore-Statistics-2018.pdf.

World Bank \& Energy Sector Management Assistance Program (2020). Offshore wind technical potential. Maps. https://esmap.org/node/197070.

WORLD BANK (2021). Renewable energy consumption. https://data.worldbank.org/indicator/ EG.FEC.RNEW.ZS.

WorLD BANK (2019). Going Global: Expanding offshore wind to emerging markets. Washington D.C.: World Bank Group. http://documents.worldbank.org/curated/ en/716891572457609829/Going-Global-Expanding-Offshore-Wind-To-Emerging-Markets.

\section{Normativa citada}

Acuerdo de París. 12 de diciembre de 2015.

Unión Europea. Comisión Europea (2020a). Una estrategia de la UE para aprovechar el potencial de la energía renovable marina para un futuro climáticamente neutro, 19 de noviembre de 2020, COM (2020) 741 final.

Unión Europea. Comisión Europea (2020b). Intensificar la ambición climática de Europa para 2030: Invertir en un futuro climáticamente neutro en beneficios de nuestros ciudadanos, 19 de septiembre de 2020, COM (2020) 562 final.

Unión Europea. Comisión Europea (2019). El Pacto Verde Europeo, 11 de diciembre de 2019, COM (2019) 640 final.

Unión Europea. Comisión Europea (2014). La energía azul. Medidas necesarias para aprovechar el potencial de la energía oceánica de los mares y océanos europeos hasta 2020 y en adelante, 20 de enero de 2014, COM (2014) 8 final.

Unión Europea. Comisión Europea (2012). Crecimiento azul. Oportunidades para un crecimiento marino y marítimo sostenible. COM (2012) 494 final. 
Unión Europea. Comisión Europea (2010). Las prioridades de la infraestructura energética a partir de 2020 - Esquema para una red de energía europea integrada, 17 de noviembre de 2010, COM (2010) 977 final.

Unión Europea. Comisión Europea (2008). Energía eólica marina: Acciones necesarias para alcanzar los objetivos de política energética para el año 2020 y los años posteriores, 12 de diciembre de 2008. COM (2008) 768 final.

European Commission (2018). A Clean Planet for all. A European strategic long-term vision for a prosperous, modern, competitive and climate neutral economy, 28 de noviembre de 2018, COM (2018) 773 final.

Ministerio para la Transición Ecológica y el reto Demográfico (2021). Resolución de la Dirección General de Política Energética y Minas por la que se resuelve la primera subasta celebrada para el otorgamiento del régimen económico de energías renovables al amparo de lo dispuesto en la orden TED/1161/2020, de 4 de diciembre. Disponible en: https://energia.gob.es/electricidad/energias-renovables/convocatorias/Documents/ Resolucionsubasta_26012021.pdf.

Parlamento y Consejo Europeos (2014). Directiva 2014/89/UE, por la que se establece un marco para la ordenación del espacio marítimo. DOUE L 257/135, 23 de julio. 